Der Bericht des Rechtsausschusses gliederte sich in zwölf Unterpunkte, die der Berichterstatter verfasst und dem Ausschuss zur Abstimmung vorgelegt hatte. Einer wurde einstimmig verabschiedet, alle übrigen wurden von der Mehrheit aus SPD und FDP beschlossen.

Auf der Basis dieser Parlamentsdrucksachen wurden am 17. Mai 1972 die Verträge mit Mehrheit im Plenum beschlossen. Ein großer Teil der CDU/CSU-Fraktion enthielt sich dabei der Stimme. Das Protokoll dieser Plenarsitzung enthält die größte Zahl Persönlicher Bemerkungen, die in der Geschichte des Bundestages von einzelnen Abgeordneten zu einzelnen Abstimmungen abgegeben wurden. Eine ganze Anzahl von ihnen ist sogar in der internationalen Presse nachgedruckt und kommentiert worden. Meine Bemerkungen als Berichterstatter wurden zum Beispiel von fast der gesamten polnischen Presse im Wortlaut wiedergegeben. Der historische Rang dieser Abstimmung ließ es zudem als geboten erscheinen, die Arbeit des Rechtsausschusses an diesen Verträgen international breiter bekannt zu machen. Dem dienten Vorträge vor auswärtigen Gesellschaften in Wien, Paris, Kopenhagen und Oslo, die zum Teil in den außenpolitischen Zeitschriften der entsprechenden Staaten nachgedruckt wurden. ${ }^{4}$ In der sowjetischen Zeitung „Prawda“ wurde seinerzeit ein Interview mit dem Berichterstatter speziell über die laufende Arbeit des Rechtsausschusses an den Ostverträgen gedruckt. ${ }^{5}$ Und heute, fast genau 40 Jahre nach der Abstimmung im Deutschen Bundestag, gilt es daran zu erinnern, welchen Anteil das Parlament und seine Arbeitsstrukturen am Gelingen dieses für die deutsche Geschichte so bedeutenden Vertragswerks gehabt haben.

4 Vgl. Claus Arndt, Rechtliche und politische Aspekte der deutschen Ostpolitik, in: Österreichische Zeitschrift für Außenpolitik, 14. Jg. (1974), H. 6, S. 395 ff.; ders., Les aspects juridiques et politiques de la „Ostpolitik“ allemande de 1970 à 1976, in: Politique Etrangère, 41. Jg. (1976), H. 3, S. 269 - 279; ders., De politiske og retslige sider af Tysklands „Ostpolitik“ 1970/76, in: Fremtiden, 31. Jg. (1976), H. 4, S. 24 ff.; ders., Legal Problems of the German Eastern Treaties, in: American Journal of International Law, 74. Jg. (1980), H. 1, S. $122-133$.

5 In völliger Übereinstimmung mit den Rechtsnormen, Interview in: Prawda (Moskau) vom 8. März 1972, S. 4.

\title{
Wie sehen Parlamentarier den Einfluss der Medien? Ergebnisse einer Befragung der Bundestagsabgeordneten*
}

\author{
Marco Dohle, Christoph Blank und Gerhard Vowe
}

Wie die Auseinandersetzungen vor und nach Rücktritten von Spitzenpolitikern zeigen, wird die Bedeutung von Medien für den Prozess der politischen Willensbildung und Entscheidungsfindung außerhalb und innerhalb der Wissenschaft intensiv diskutiert. Politik, so eine häufig getätigte Aussage, orientiere sich in starkem und zunehmendem Maße an medialen Zeithorizonten, Selektionsregeln und Rollenvorgaben. ${ }^{1}$ Um diese Orientierung an den Me-

* Wir danken der Redaktion der Zeitschrift für Parlamentsfragen für die wertvollen Hinweise, die wir zu einer ersten Version des vorliegenden Textes erhalten haben. 
dien zu untersuchen und zu erklären, wird zumeist versucht, die Aktivitäten von Politikern zu erfassen: So wurde zum Beispiel die Anzahl eher öffentlichkeitsorientierter Tätigkeiten (zum Beispiel Anhörungen, Aktuelle Stunden) mit der Anzahl gesetzgeberischer Aktivitäten im Deutschen Bundestag im Zeitverlauf verglichen. ${ }^{2}$ Oder es wurde ermittelt, in welchem Ausmaß Abgeordnete des Bundestages in ihren Reden auf mediale Quellen verwiesen, um ihre Aussagen argumentativ zu untermauern. ${ }^{3}$

Einen anderen Weg schlagen Studien ein, in denen politische Akteure entweder danach befragt werden, wie sie den Einfluss von Medien auf die Politik insgesamt beurteilen ${ }^{4}$, oder wie sie den Medieneinfluss auf die eigene Person oder auf andere Personengruppen einschätzen: Letzteres gibt unter anderem Aufschluss darüber, ob die wahrgenommene Bedeutung der Medien Folgen für die Einstellungen der Politiker hat. So liegt zum Beispiel die Vermutung nahe, dass Politiker, die den Medien eine zu große politische Beeinflussung der Bevölkerung zuschreiben, dann auch der Ansicht sind, dass dieser Einfluss begrenzt werden sollte. Die Prüfung solcher Annahmen war das Ziel einer Studie unter Abgeordneten des 16. Deutschen Bundestages. Die Untersuchung gibt eine Antwort auf folgende Fragen: Wie stark schätzen Bundestagsabgeordnete den politischen Einfluss von Medien ein, und zwar auf sich selbst und auf andere? Und welche Folgen haben diese Einschätzungen auf ihre Meinungen dazu, ob der Medieneinfluss möglicherweise eingeschränkt gehört?

\section{Die Wahrnehmung von Medieneinflüssen im politischen Kontext}

Die Unterscheidung zwischen dem Einfluss auf sich selbst und dem auf andere verweist auf die theoretische Grundlage der Studie, nämlich auf Ansätze zu indirekten Medienwirkungen wie den Third-Person-Effekt ${ }^{5}$ sowie den Influence-of-Presumed-Media-Influence-Approach. ${ }^{6}$ Diesen Ansätzen zufolge sind Einflussvermutungen deshalb relevant, weil sie auf Einstellungen oder Handlungsweisen von Personen wirken - selbst wenn diese Vermutungen

1 Häufig wird dies als Mediatisierung beziehungsweise Medialisierung der Politik bezeichnet. Vgl. Gianpietro Mazzoleni / Winfried Schulz, „Mediatization” of Politics. A Challenge for Democracy?, in: Political Communication, 16. Jg. (1999), H. 3, S. 247 - 261; Gerhard Vowe, Mediatisierung der Politik? Ein theoretischer Ansatz auf dem Prüfstand, in: Publizistik, 51. Jg. (2006), H. 4, S. 437 - 455; Jesper Strömbeck, Four Phases of Mediatization: An Analysis of the Mediatization of Politics, in: International Journal of Press/Politics, 13. Jg. (2008), H. 3, S. 228 - 246; Carsten Reinemann, Medialisierung ohne Ende? Zum Stand der Debatte um Medieneinflüsse auf die Politik, in: ZPol, 57. Jg. (2010), H. 3, S. 278 - 293.

2 Vgl. Hans Mathias Kepplinger, Mediatization of Politics. Theory and Data, in: Journal of Communication, 52. Jg. (2002), H. 4, S. $972-986$.

3 Vgl. Gerhard Vowe / Marco Dohle, Weltsicht und Medienbild des Parlaments im Wandel. Eine Inhaltsanalyse von Bundestagsdebatten aus 50 Jahren, in: Frank Marcinkowski / Barbara Pfetsch (Hrsg.), Politik in der Mediendemokratie, PVS-Sonderheft 42, Wiesbaden 2009, S. 224 - 250.

4 Vgl. Hans Mathias Kepplinger, Rivalen um Macht und Moral: Bundestagsabgeordnete und Hauptstadtjournalisten, in: Hanna Kaspar / Harald Schoen / Siegfried Schumann / Jürgen R. Winkler (Hrsg.), Politik - Wissenschaft - Medien, Wiesbaden 2009, S. 307 - 321; Peter Maurer, Explaining Perceived Media Influence in Politics. An Analysis of the Interplay of Context and Attitudes in Four European Democracies, in: Publizistik, 56. Jg. (2011), H. 1, S. 27 - 50.

5 Vgl. W. Phillips Davison, The Third-Person Effect in Communication, in: Public Opinion Quarterly, 47. Jg. (1983), H. 1, S. 1 - 15.

6 Vgl. Albert C. Gunther / J. Douglas Storey, The Influence of Presumed Influence, in: Journal of Communication, 53. Jg. (2003), H. 2, S. $199-215$. 
gegenüber der Realität verzerrt sein mögen. ${ }^{7}$ Die zentralen Annahmen des Third-PersonEffekts sind: (1) Individuen sind in der Regel der Auffassung, dass andere stärker als sie selbst (als sogenannte „first person“) von Medien beeinflusst werden. Die anonyme Allgemeinheit, die „third persons“, wird dabei als noch beeinflussbarer angesehen als Personen aus dem unmittelbaren Umfeld, die „second persons“ (Wahrnehmungskomponente/Third-PersonPerception). (2) Diese Wahrnehmungsdifferenz - vor allem diejenige zwischen erster Person und dritten Personen - hat Konsequenzen für Vor- und Einstellungen beziehungsweise für Verhaltensabsichten und Verhalten (Verhaltenskomponente/Third-Person-Behavior). Weiter greift der Influence-of-Presumed-Media-Influence-Approach aus: Diesem Ansatz zufolge werden eigene Einstellungen und eigenes Verhalten maßgeblich bereits davon beeinflusst, welche Wirkung Individuen den Medien auf andere Rezipienten zuschreiben - ohne dass dabei eine Differenz zwischen dem angenommenen Einfluss auf die eigene Person und jenem auf andere Personengruppen eine Rolle spielt.

Die Annahmen zur Wahrnehmungsdifferenz sind durch die empirische Forschung gut bestätigt. ${ }^{8}$ Im Hinblick auf die Folgen der Einflusswahrnehmungen sind die Befunde widersprüchlicher. In einigen Studien konnten Auswirkungen auf Vor- und Einstellungen sowie Verhalten gemessen werden, in anderen nicht. Die Stärke dieser Konsequenzen variiert beispielsweise je nach abhängiger Variable: So wurde insbesondere mehrfach bestätigt, dass Personen der Forderung nach einer Zensur von aus ihrer Sicht bedenklichen Medieninhalten zustimmen, wenn sie diesen eine hohe Wirkung auf andere Personen beziehungsweise eine höhere Wirkung auf andere als auf sich selbst zuschreiben. ${ }^{9}$

Eine Vielzahl von Third-Person-Studien im Zusammenhang mit politischen Medieninhalten befasste sich mit der Wahrnehmung des Einflusses der Medienberichterstattung in Wahlkämpfen ${ }^{10}$, politischer Werbung ${ }^{11}$ oder der Veröffentlichung von politischen Meinungsumfragen. ${ }^{12}$ Auch in diesen Untersuchungen wurden Differenzen zwischen dem zugeschriebenen Einfluss auf die eigene Person und dem auf dritte Personen ermittelt. Auswirkungen der Wahrnehmungsdifferenzen ergaben sich ebenfalls partiell: Beim

7 Die Theorien basieren unter anderem auf dem Thomas-Theorem als basalem sozialwissenschaftlichen Ansatz, wonach auch subjektive Annahmen über soziale Tatsachen folgenreiche soziale Tatsachen sind; vgl. William I. Thomas / Dorothy S. Thomas, The Child in America: Behavior Problems and Programs, New York 1928.

8 Vgl. Ye Sun / Zhongdang Pan / Lijiang Shen, Understanding the Third-Person Perception: Evidence from a Meta-Analysis, in: Journal of Communication, 58. Jg. (2008), H. 2, S. $280-300$.

9 Für aktuelle Überblicke siehe unter anderem Richard M. Perloff, Mass Media, Social Perception, and the Third-Person Effect, in: Jennings Bryant / Mary Beth Oliver (Hrsg.), Media Effects. Advances in Theory and Research, New York 2009, S. 252 - 268; Nurit Tal-Or / Yariv Tsfati / Albert C. Gunther, The Influence of Presumed Media Influence. Origins and Implications of the ThirdPerson Perception, in: Robin L. Nabi / Mary Beth Oliver (Hrsg.), The SAGE Handbook of Media Processes and Effects, Thousand Oaks 2009, S. 99 - 112.

$10 \mathrm{Vgl}$. Antonis Gardikiotis, Group Distinctiveness, Political Identification, and the Third-Person Effect: Perceptions of a Political Campaign in the 2004 Greek National Election, in: Media Psychology, 11. Jg. (2008), H. 3, S. $331-353$.

11 Vgl. Patrick C. Meirick, Topic-Relevant Reference Groups and Dimensions of Distance. Political Advertising and First- and Third-Person Effects, in: Communication Research, 31. Jg. (2004), H. 2, S. $234-255$.

12 Vgl. Vincent Price / Natalie Jomini Stroud, Public Attitudes Toward Polls: Evidence from the 2000 US Presidential Election, in: International Journal of Public Opinion Research, 18. Jg. (2006), H. 4, S. $393-421$. 
Befürwortungsgrad von Zensurmaßnahmen erwies sich die Wahrnehmungsdifferenz als relevant ${ }^{13}$, für andere Folgen wie etwa größere Anstrengungen bei der eigenen politischen Meinungsbildung ${ }^{14}$ jedoch nicht immer. Deutlichere Befunde zeitigten Untersuchungen, die nicht auf die Wahrnehmungsdifferenzen gerichtet waren, sondern auf vermutete Wirkungen auf andere. So konnte zum Beispiel ermittelt werden, dass ein angenommener Medieneinfluss auf die Entscheidungen der anderen Wähler Auswirkungen auf das eigene, von strategischen Überlegungen mitgeprägte Wahlverhalten der Befragten hatte. ${ }^{15}$

In den Untersuchungen zum Third-Person-Effekt oder Influence-of-Presumed-MediaInfluence-Approach, die in einem politischen Kontext standen, wurden im Regelfall Bürger befragt. Bei Politikern zeigt sich dagegen ein anderes Bild: Einerseits liegt eine Reihe aussagekräftiger Studien vor, in denen sie etwa zu ihrer eigenen Medienaffinität, ihrer Beurteilung der medialen Berichterstattung sowie ihrer Einschätzung des Einflusses von Medien auf die Politik insgesamt Auskunft erteilten. ${ }^{16}$ Eine Befragung unter Abgeordneten des Deutschen Bundestages ergab etwa, dass diese den Medieneinfluss auf die Politik als sehr stark empfinden und ihn gerade deshalb skeptisch betrachten. Ihren eigenen Einfluss auf die Medien schätzen die Abgeordneten dagegen als spürbar geringer ein. ${ }^{17}$ Eine Untersuchung bei Parlamentsabgeordneten aus Belgien und den Niederlanden kam zu einem ähnlichen Ergebnis: Der mediale Einfluss auf die Politik wird als groß eingeschätzt und von vielen Politikern kritisch als zu groß eingestuft. ${ }^{18}$

Andererseits gibt es nur sehr wenige Studien, in denen politische Akteure zu ihrer Wahrnehmung des politischen Medieneinflusses auf unterschiedliche Personen(gruppen) - darunter auch die eigene Person - befragt und zudem mögliche Konsequenzen dieser Wahrneh-

13 Vgl. Michael B. Salwen, Perceptions of Media Influence and Support for Censorship. The ThirdPerson Effect in the 1996 Presidential Election, in: Communication Research, 25. Jg. (1998), H. 3, S. $259-285$.

14 Vgl. Patrick Rössler, Wie Menschen die Wirkungen politischer Medienberichterstattung wahrnehmen - und welche Konsequenzen daraus resultieren. Zum Zusammenhang von politischer Willensbildung, Second- und Third-Person-Effekten, in: Frank Marcinkowski / Barbara Pfetsch (Hrsg.), a.a.O. (Fn. 3), S. $468-495$.

15 Vgl. Jonathan Cohen / Yariv Tsfati, The Influence of Presumed Media Influence in Strategic Voting, in: Communication Research, 36. Jg. (2009), H. 3, S. 359 - 378.

16 Beispiele für aktuelle Untersuchungen sind Hans Mathias Kepplinger, a.a.O. (Fn. 4); Dorothee Marx, Landtagsabgeordnete im Fokus der Medien. Ihre Sicht auf Entstehung, Wirkung und Qualität landespolitischer Berichterstattung, Baden-Baden 2009; Peter Maurer, a.a.O. (Fn. 4); Barbara Pfetsch / Eva Mayerhöffer, Vordergründige Nähe. Zur Kommunikationskultur von Politik- und Medieneliten in Deutschland, in: Medien \& Kommunikationswissenschaft, 59. Jg. (2011), H. 1, S. 40 - 59; Daniel Pontzen, Nur Bild, BamS und Glotze? Medialisierung der Politik aus Sicht der Akteure, Münster 2006; Jesper Strömbäck / Lars W. Nord, Do Politicians Lead the Tango? A Study of the Relationship between Swedish Journalists and their Political Sources in the Context of Election Campaigns, in: European Journal of Communication, 21. Jg. (2006), H. 2, S. 147 - 164; Jens Tenscher, Salto mediale? Medialisierung aus der Perspektive deutscher Landtagsabgeordneter, in: Michael Edinger / Werner J. Patzelt (Hrsg.), Politik als Beruf, PVS-Sonderheft 44, Wiesbaden 2011, S. 375 - 395; Peter van Aelst / Kees Brants / Philip van Praag / Claes de Vreese / Michiel Nuytemans / Arjen van Dalen, The Fourth Estate as Superpower? An Empirical Study of Perceptions of Media Power in Belgium and the Netherlands, in: Journalism Studies, 9. Jg. (2008), H. 4, S. $494-511$.

17 Vgl. Hans Mathias Kepplinger, a.a.O. (Fn. 4).

18 Vgl. Peter van Aelst / Kees Brants / Philip van Praag / Claes de Vreese / Michiel Nuytemans / Arjen van Dalen, a.a.O. (Fn. 16). 
mungen berücksichtigt wurden. Eine davon wurde unter Knesset-Abgeordneten in Israel durchgeführt ${ }^{19}$ : Sie sollten darlegen, wie stark sie den Einfluss der Berichterstattung israelischer Medien auf die Wähler im eigenen Land sowie auf Abgeordnetenkollegen einschätzten. Zugleich wurden die Anzahl ihrer mediengerechten und öffentlichkeitswirksamen Tätigkeiten sowie deren Anteil an ihrem politischen Wirken gemessen. Vermutungen zur medialen Beeinflussbarkeit ihrer Kollegen haben, so die Ergebnisse, keine signifikante Bedeutung für das eigene Handeln. Die Annahme hoher Medieneinflüsse auf die Wähler führte aber bei den Parlamentariern in der Tat dazu, sich verstärkt mediengerecht zu betätigen. Dieses Resultat bestätigt Vermutungen, wonach der Ausgangspunkt einer verstärkten Medienorientierung von Politikern nicht unbedingt „ein tatsächlicher Wandel von Medien oder Medieneinflüssen, sondern zunächst einmal die veränderte Wahrnehmung von Medieneinflüssen " ${ }^{20}$ sei. Politiker richten demnach vor allem deshalb ihr politisches Handeln nach den Vorgaben der Medien aus, weil sie glauben, dass diese einen enormen Einfluss auf die Wählerschaft ausüben.

Es ist also davon auszugehen, dass Politiker im Regelfalle die Allgemeinheit für stark beeinflussbar durch Medien halten. Vermutet werden kann zudem, dass sie sich selbst für wenig beeinflussbar halten, weil sie sich höhere politische und mediale Kompetenz zusprechen als anderen Personen. ${ }^{21}$ Diese Annahme konnte durch die Ergebnisse einer ThirdPerson-Befragung unter Politikern, Journalisten und Bürgern in Deutschland untermauert werden: Politiker empfinden sich selbst im Vergleich zu Journalisten und Bürgern nur in geringem Maße durch Medien geprägt. ${ }^{22}$ Die Studie verdeutlichte außerdem eine mögliche Konsequenz wahrgenommener Medieneinflüsse. Je stärker die politisch Aktiven mediale Wirkungen auf die Allgemeinheit vermuten, desto eher stimmen sie tendenziell der Forderung zu, den Einfluss von Medien auf die Politik einzuschränken. ${ }^{23}$ Diese Folge von Einflusswahrnehmungen auf andere Personen ist auch deshalb relevant, weil Politiker eher Einfluss auf Medien nehmen können, als dies einfache Bürger vermögen; Politiker könnten beispielsweise eine Verschärfung der Vorschriften für Medien, die ihnen aufgrund vermuteter Einflüsse als notwendig erscheint, nicht nur fordern, sondern auch fördern. Dies gilt umso mehr für Abgeordnete.

19 Vgl. Jonathan Cohen / Yariv Tsfati / Tamir Sheafer, The Influence of Presumed Media Influence in Politics. Do Politicians' Perceptions of Media Power Matter?, in: Public Opinion Quarterly, 72. Jg. (2008), H. 2, S. 331 - 344. Ein weiteres Beispiel ist die Studie von Dorothee Marx, a.a.O. (Fn. 16). Dort wurden Landtagsabgeordnete gebeten, die Wirkung von Medienberichten auf unterschiedliche Personengruppen einzuschätzen. Es zeigten sich dabei leichte Zusammenhänge zwischen Wirkungseinschätzungen und eigener Mediennutzung. Andere mögliche Konsequenzen von Einflusswahrnehmungen wurden jedoch nicht erfasst.

20 Carsten Reinemann, a.a.O. (Fn. 1), S. 285.

21 Vgl. Dominic L. Lasorsa, Policy Makers and the Third-Person Effect, in: J. David Kennamer (Hrsg.), Public Opinion, the Press and Public Policy, Westport 1992, S. 163 - 175.

22 Vgl. Marco Dohle / Gerhard Vowe, Wahrnehmung der politischen Einflüsse von Medienangeboten. Third-Person-Effekte bei Bürgern, Journalisten und Politikern im Vergleich, in: Christian Schemer / Werner Wirth / Carsten Wünsch (Hrsg.), Politische Kommunikation: Wahrnehmung, Verarbeitung, Wirkung, Baden-Baden 2010, S. 11 - 29. Die Studie beruhte allerdings nicht auf repräsentativen Stichproben. Für die Gruppe der befragten Politiker wurde auf in Parteien aktive Personen zurückgegriffen, auf Personen in politischen Ämtern, aber auch auf Personen, die in Gewerkschaften sozial- und gesellschaftspolitische Aufgaben erfüllten.

23 Die Annahme eines starken Einflusses auf sich selbst sowie Wahrnehmungsdifferenzen im Sinne der Third-Person-Perception waren dagegen für die Intensität dieser Forderung folgenlos. 
Insgesamt liegen also einige empirische Hinweise auf die Relevanz der Einflusswahrnehmung durch Politiker vor; sie bleiben aber eher punktuell. Deshalb wurde in einer Befragung von Abgeordneten des Deutschen Bundestages systematisch geprüft, welche Beeinflussung durch Medien sie verschiedenen Personen(gruppen) unterstellen, ob sie einen starken Medieneinfluss kritisch bewerten und ob sich daraus Forderungen nach einer Einschränkung dieses Einflusses ergeben. Grundlage bildeten die folgenden Annahmen:

(1) Die Bundestagsabgeordneten nehmen an, dass ihr unmittelbares Umfeld (second persons) stärker als sie selbst (first person) durch die Medien politisch beeinflusst wird; sie nehmen außerdem an, dass die anonyme Allgemeinheit (third persons) noch stärker als ihr unmittelbares Umfeld durch die Medien politisch beeinflusst wird.

(2) Die kritische Haltung von Politikern gegenüber einem starken Medieneinfluss auf die Politik insgesamt überträgt sich darauf, wie sie den Einfluss auf die eigene Person und auf andere Personen beurteilen: Je stärker die Bundestagsabgeordneten den Einfluss der Medien auf die eigene Person und auf andere Personen einschätzen, desto negativer werden sie ihn beurteilen.

(3) Mit zunehmender Wahrnehmung eines starken Medieneinflusses auf andere Personen steigt die Zustimmung der Bundestagsabgeordneten zu der Forderung nach einer Einschränkung dieses Einflusses.

\section{Empirische Befunde zur Wahrnehmung des Medieneinflusses}

\subsection{Methodische Umsetzung}

Um diese Annahmen zu prüfen, wurde im Frühjahr 2009 eine standardisierte Befragung unter den 612 Abgeordneten des 16. Deutschen Bundestages durchgeführt. In einem ersten Schritt wurden die gedruckten Fragebögen über vorher kontaktierte Mitglieder der verschiedenen Fraktionen an die Abgeordneten verteilt. Ausgefüllte Fragebögen wurden in anonymisierter Form über diese „Mittelsfrauen“ und „Mittelsmänner“ an die Verantwortlichen der Studie zurückgesandt. In einem zweiten Schritt wurden alle Mitglieder des Bundestages per E-Mail um die Teilnahme an der Untersuchung gebeten. Der Fragebogen war der Einladung als Dokument angehängt und konnte in ausgedruckter Form ausgefüllt und anonymisiert zurückgesandt werden.

Er beinhaltete unter anderem Items, mit denen der Einfluss der Medien eingeschätzt werden sollte. Differenziert wurde in Einflusszuschreibungen auf die eigene Person (first person) ${ }^{24}$, auf Personen in der näheren persönlichen Umgebung (second persons) ${ }^{25}$ sowie auf die Gesamtbevölkerung (third persons). ${ }^{26}$ Auf die Frage nach vermuteten Einflüssen auf andere Politiker wurde verzichtet. Dies hätte es nötig gemacht, eine Reihe von Differenzierungen (zum Beispiel andere Bundestagsabgeordnete generell, Abgeordnete der eigenen

24 Itemformulierung: „Mein Bild von der Politik wird stark von den Berichten in den Medien beeinflusst."

25 Itemformulierung: „Das Bild von der Politik wird bei Menschen in meinem Freundes- und Bekanntenkreis stark von den Berichten in den Medien beeinflusst.“

26 Itemformulierung: „Das Bild von der Politik wird bei der Allgemeinheit der Bevölkerung stark von den Berichten in den Medien beeinflusst." 


\begin{tabular}{|l|c|c|}
\hline Tabelle 1: Fraktionszugehörigkeit der Stichprobe und der Grundgesamtheit (in Prozent) \\
\hline & Befragung $(\mathrm{N}=208)^{*}$ & Grundgesamtheit $(\mathrm{N}=612)$ \\
\hline CDU/CSU & 51,7 & 36,4 \\
SPD & 19,5 & 36,3 \\
FDP & 14,1 & 10,0 \\
Bündnis 90/Die Grünen & 6,8 & 8,3 \\
Die Linke & 7,8 & 8,7 \\
Fraktionslos & - & 0,3 \\
\hline * Drei Personen ohne Angabe. & \\
Quelle: Eigene Erhebung. & \\
\hline
\end{tabular}

Fraktion, Abgeordnete anderer Fraktionen) vorzunehmen, was die Befragung monoton und zeitraubend gemacht hätte. Außerdem sollten die Befragten die Bedeutung von Medien beurteilen ${ }^{27}$ und einer Aussage zustimmen oder sie ablehnen, in der eine mögliche Begrenzung des Medieneinflusses thematisiert wurde. ${ }^{28}$ Mit weiteren Items wurden soziodemografische Variablen, die Fraktionszugehörigkeit und die Mandatsdauer erhoben. ${ }^{29}$

An der Studie beteiligten sich 208 der 612 Abgeordneten (34,0 Prozent), ein üblicher Wert für Befragungen unter Mitgliedern des Bundestages. ${ }^{30} 67$ Befragte waren weiblich (33,0 Prozent), 136 männlich (67,0 Prozent; fünf ohne Geschlechtsangabe); dies entspricht der Geschlechterverteilung aller Bundestagsabgeordneten zum Zeitpunkt der Erhebung (32,4 Prozent weiblich; 67,6 Prozent männlich).

Verzerrt ist die Stichprobe allerdings im Hinblick auf die Fraktionszugehörigkeit (Tabelle 1). Neben kleineren Abweichungen bei den anderen Fraktionen fallen die stark überdurchschnittliche Beteiligung von Abgeordneten der CDU/CSU-Fraktion und die stark unterdurchschnittliche Teilnahmebereitschaft der SPD-Abgeordneten auf. ${ }^{31}$

27 Itemformulierung: „Die Medien haben bei uns einen zu großen Einfluss.“

28 Itemformulierung: „Der Einfluss der Medien auf die Politik sollte eingeschränkt werden, zum Beispiel durch Vorschriften, sachlicher zu berichten."

29 Ein weiterer Teil des Instruments umfasste Fragen zur Kommunikation der Abgeordneten mit den Bürgern - dies stand im Kontext einer anderen Untersuchung.

30 Vgl. Bernhard Weßels, Abgeordnetenbefragung 2003. Kurzfassung und Dokumentation der Ergebnisse, Berlin 2003; http://www.wz-berlin.de/zkd/dsl/download.de.htm (Abruf am 14. Mai 2012); Hans Mathias Kepplinger, a.a.O. (Fn. 4); Jens Tenscher / Laura Will, Abgeordnete online? Internetaktivitäten und -bewertungen der Mitglieder des Deutschen Bundestages, in: ZParl, 41. Jg. (2010), H. 3, S. $504-518$.

31 Da die Stichprobe (entstanden aus einer Vollerhebung) in zumindest diesem Merkmal kein repräsentatives Abbild der Grundgesamtheit ist, sind Rückschlüsse auf eben diese Grundgesamtheit mit großer Vorsicht zu behandeln. Dennoch werden im Folgenden auch Ergebnisse von Signifikanztests aufgeführt. In den zugrunde liegenden Berechnungen wurde dabei die Parteizugehörigkeit auf unterschiedliche Weise kontrolliert (siehe die Fußnoten 32, 36 und 39). Auf Gewichtungen der Daten wurde verzichtet. Vgl. dazu zum Beispiel Christian Alt / Walter Bien, Gewichtung, ein sinnvolles Verfahren in der Sozialwissenschaft?, in: Siegfried Gabler / Jürgen H. P. Hoffmeyer-Zlotnik I Dagmar Krebs (Hrsg.), Gewichtung in der Umfragepraxis, Opladen 1994, S. $124-140$. 


\section{Abbildung 1: Von Bundestagsabgeordneten wahrgenommene Medieneinflüsse (Mittelwerte)}

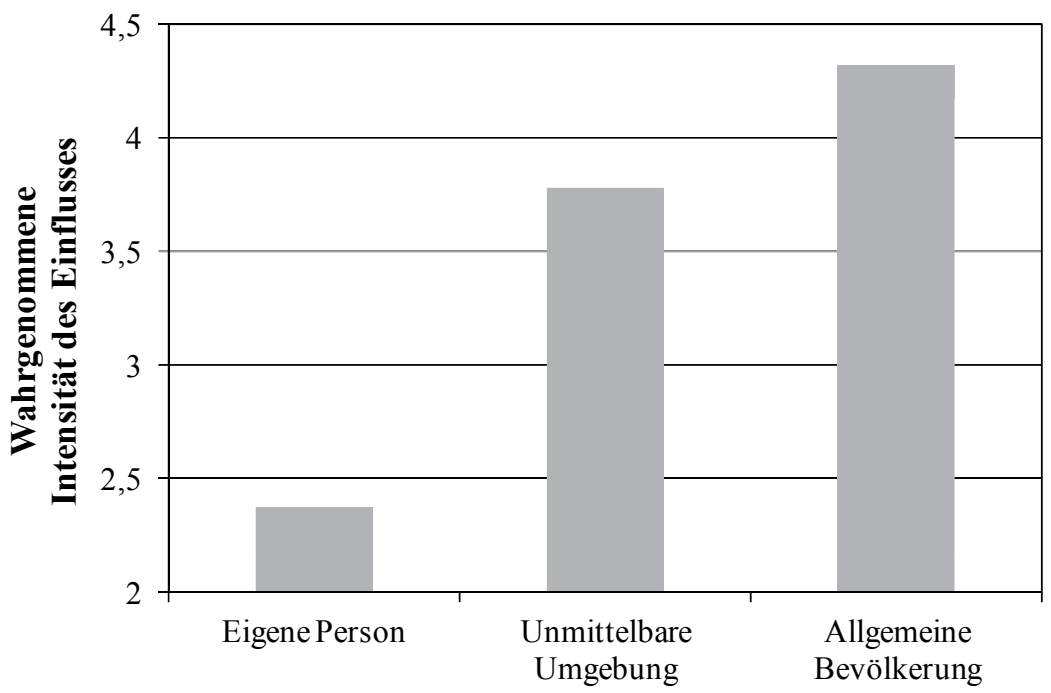

Wahrgenommener Einfluss auf ...

Anmerkung: Antwortvorgaben jeweils von 1 (,stimme überhaupt nicht zu“) bis 5 („stimme voll und ganz $\mathrm{zu}^{\prime \prime)}$.

Quelle: Eigene Erhebung.

\subsection{Ergebnisse}

Die Ergebnisse sind nach den drei leitenden Annahmen geordnet. Die erste Vermutung war, dass die Bundestagsabgeordneten den politischen Einfluss der Medien auf sich selbst im Vergleich zum Einfluss auf Personen in ihrer unmittelbaren Umgebung als geringer einschätzen würden. Die stärksten Einflusszuschreibungen wurden im Hinblick auf die Allgemeinheit erwartet. Die Ergebnisse bestätigen dies (siehe Abbildung 1). Der Einfluss auf die eigene Person wird als recht gering eingestuft (Mittelwert: 2,37; Standardabweichung: 1,07) - er liegt deutlich unter dem Skalenmittelpunkt. Sehr viel höher ist nach Einschätzung der Abgeordneten der Einfluss auf ihre unmittelbare Umgebung (Mittelwert: 3,78; Standardabweichung: 0,88). Die allgemeine Bevölkerung wird als am stärksten medial beeinflussbar angesehen (Mittelwert: 4,32; Standardabweichung: 0,64). Der zugeschriebene Einfluss auf die Gesamtbevölkerung ist somit nicht weit von Skalenendpunkt entfernt. ${ }^{32}$ Auffällig an den

32 Zusätzlich wurden Varianzanalysen mit Messwiederholung gerechnet. In die Analysen wurde die Fraktionszugehörigkeit auf zwei Arten als Kovariate aufgenommen, um die Verzerrungen in der Stichprobe zugunsten der CDU/CSU beziehungsweise der bürgerlichen Parteien CDU/CSU und FDP zu berücksichtigen (Version A: "0" = CDU/CSU; „1" = übrige Parteien; Version B: „0" = bürgerliche Parteien; „““ = linke Parteien). In beiden Analysen ergab sich ein signifikanter Gesamteffekt (Version A: $F(1.62,197)=176,01, p<.001 ; \eta^{2}=0,47$; Version B: $F(1.62,197)$ $=215,93, p<.001 ; \eta^{2}=0,52$; wegen ungleicher Varianzen zwischen den Items wurde eine 


\begin{tabular}{|l|c|c|c|}
\hline Tabelle 2: & Wahrgenommene Medieneinflüsse getrennt nach Fraktionszugehörigkeit (Mittelwerte) \\
\hline & $\begin{array}{c}\text { Auf eigene } \\
\text { Person }\end{array}$ & $\begin{array}{c}\text { Auf die unmittelbare } \\
\text { Umgebung }\end{array}$ & $\begin{array}{c}\text { Auf die allgemeine } \\
\text { Bevölkerung } \\
\varnothing(s)\end{array}$ \\
\hline CDU/CSU & $\varnothing(s)$ & $4,36(0,64)$ \\
SPD & $2,43(1,03)$ & $3,86(0,86)$ & $4,26(0,64)$ \\
FDP & $2,26(1,16)$ & $3,79(1,01)$ & $4,21(0,74)$ \\
Bündnis 90/Die Grünen & $2,41(1,15)$ & $3,71(0,94)$ & $4,31(0,48)$ \\
Die Linke & $2,58(1,00)$ & $3,58(0,67)$ & $4,27(0,59)$ \\
\hline Anmerkung: Ausgewiesen sind die jeweiligen Mittelwerte (Ø) und Standardabweichungen (s); Antwort- \\
vorgaben jeweils von 1 (,stimme überhaupt nicht zu“) bis 5 (,stimme voll und ganz zu“). \\
Quelle: Eigene Erhebung.
\end{tabular}

Werten ist angesichts des bisherigen Forschungsstandes weniger, dass es zu Wahrnehmungsdifferenzen in der vorgefundenen Richtung kam. Bemerkenswert ist aber die sehr hohe Differenz zwischen den Werten. ${ }^{33}$

Die Einschätzungen zum Medieneinfluss wurden zusätzlich für verschiedene Gruppen getrennt gerechnet. Die Unterschiede sind jeweils gering. Dies gilt für den Vergleich weiblicher und männlicher Abgeordneter wie für jüngere und ältere Parlamentarier. Auch die Mandatsdauer hat keinen deutlichen Einfluss auf die Angaben.

Ebenfalls gering sind die Unterschiede zwischen den Fraktionen (vgl. Tabelle 2). Die Mittelwerte für den wahrgenommenen Einfluss auf die allgemeine Bevölkerung schwanken zum Beispiel zwischen 4,26 und 4,36. ${ }^{34}$ Etwas größer fallen die Unterschiede bei der Selbsteinschätzung aus: Abgeordnete von Bündnis 90/Die Grünen (Mittelwert: 2,58) sehen sich etwa als beeinflussbarer an als die Abgeordneten der Fraktion der Linken (Mittelwert: 2,13). Diese Unterschiede beruhen allerdings bei den kleinen Bundestagsfraktionen auf geringe Fallzahlen.

In einem zweiten Schritt wurden die Wirkungsvermutungen und die Bewertung des Medieneinflusses kombiniert. Der Aussage, wonach der Medieneinfluss zu groß sei, wird insgesamt tendenziell zugestimmt (Mittelwert: 3,60; Standardabweichung: 1,08; wobei 1 = „trifft überhaupt nicht zu“ bis 5 = „trifft voll und ganz zu“). Er wird demnach eher skeptisch bewertet. ${ }^{35}$

Greenhouse-Geisser-Korrektur vorgenommen). Post-Hoc-Vergleiche mit Bonferroni-Korrektur verdeutlichen zudem, dass sich auch die einzelnen Mittelwerte jeweils signifikant voneinander unterscheiden.

33 In der Untersuchung von Dohle und Vowe ergaben sich unter politisch aktiven Personen ähnliche Werte für die unmittelbare Umgebung (Mittelwert: 3,94) und die allgemeine Bevölkerung (Mittelwert: 4,44), während der Wert für die Selbsteinschätzung höher als in der vorliegenden Studie war (Mittelwert: 2,95; Skala jeweils: 1 = „trifft überhaupt nicht zu“ bis 5 = trifft voll und ganz zu“); Marco Dohle / Gerhard Vowe, a.a.O. (Fn. 22).

34 Ähnliches zeigte sich in der Landtagsstudie durch Dorothee Marx, a.a.O. (Fn. 16): Die vermutete Stärke des Medieneinflusses auf andere unterschied sich je nach Parteizugehörigkeit der Befragten nicht gravierend.

35 Getrennt nach Fraktionen: CDU/CSU - Mittelwert: 3,83; SPD - Mittelwert: 3,36; FDP - Mittelwert: 3,04; Bündnis 90/Die Grünen - Mittelwert: 3,07; Die Linke - Mittelwert: 4,13. 


\begin{tabular}{|c|c|c|c|}
\hline \multicolumn{4}{|c|}{$\begin{array}{l}\text { Lineare Regressionen zur Erklärung der Beurteilung des Medieneinflusses auf die } \\
\text { Politik }\end{array}$} \\
\hline & $b$ & $s(b)$ & $\beta$ \\
\hline Wahrgenommener Einfluss auf persönliche Umgebung & $-0,06$ & 0,08 & $-0,06$ \\
\hline Wahrgenommener Einfluss auf eigene Person & 0,08 & 0,11 & 0,06 \\
\hline Wahrgenommener Medieneinfluss auf Allgemeinheit & 0,32 & 0,14 & $0,19^{*}$ \\
\hline $\mathrm{R}^{2}$ & \multicolumn{3}{|c|}{, $05^{*}$} \\
\hline \multicolumn{4}{|c|}{$\begin{array}{l}\text { Anmerkung: Positive } b \text { - und } \beta \text {-Werte bedeuten: Je höher der jeweils wahrgenommene Einfluss, destc } \\
\text { negativer wird der Einfluss von Medien auf die Politik beurteilt; } s(b) \text { : Standardabweichung des Regressi } \\
\text { onskoeffizienten b; }{ }^{*} p<.05 ; n=197 \text {. } \\
\text { Quelle: Eigene Erhebung. }\end{array}$} \\
\hline
\end{tabular}

Die Abgeordneten, so die zweite Annahme, würden den politischen Einfluss von Medien umso negativer beurteilen, je stärker sie ihn auf ihre eigene Person und auf andere Personengruppen einschätzen. Zur Überprüfung wurde eine Regression gerechnet, in der die Beurteilung des Medieneinflusses als abhängige Variable diente. Mit diesem Verfahren kann die relative Erklärungskraft von einzelnen Faktoren auf die Beurteilung des Medieneinflusses ermittelt werden. Als unabhängige Variablen wurden die vermuteten Medieneinflüsse auf die eigene Person, auf die unmittelbare Umgebung und auf die Allgemeinheit der Bevölkerung berücksichtigt. ${ }^{36}$ Die Ergebnisse sind in Tabelle 3 dargestellt. Bei einer insgesamt geringen Erklärungskraft des Regressionsmodells hat allein der wahrgenommene Einfluss auf die allgemeine Bevölkerung eine spürbare Wirkung: Je höher er ausfällt, desto negativer wird der Einfluss von Medien auf die Politik beurteilt. Während diese Annahme also durch die Daten bestätigt wird, sind die vermuteten Einflüsse auf die eigene Person und auf die persönliche Umgebung weitgehend irrelevant.

Mit der Forderung nach einer Einschränkung des politischen Medieneinflusses wurde eine weitere Variable einbezogen. Die Aussage, den Einfluss einzuschränken, wird insgesamt eher abgelehnt (Mittelwert: 1,87; Standardabweichung: 1,09; wobei 1 = „trifft überhaupt nicht zu“ bis $5=$ "trifft voll und ganz zu“). Die Unterschiede in den Antworten sind bei einem Vergleich der Fraktionen recht beachtlich. ${ }^{37}$

Zentral war als dritte Annahme, dass eine solche Forderung umso stärker ausfallen könnte, je mehr die Abgeordneten von einem starken Medieneinfluss auf die Allgemeinheit ausgehen. Eine Regression verdeutlicht, dass diese Überlegung tendenziell durch die Daten gestützt wird $(\beta=0,13$; ns). Weiterführende Berechnungen unter zusätzlicher Berücksichtigung der Bewertung des Medieneinflusses verweisen jedoch auf einen indirekten Wirkungs-

36 Zudem wurden hierarchische Regressionen gerechnet. In diese wurden in einem ersten Block weitere Variablen integriert, die ebenfalls im Fragebogen Berücksichtigung gefunden hatten (Alter der Abgeordneten, Geschlecht der Abgeordneten, Anzahl der Wahlperioden im Bundestag, Fraktionszugehörigkeit „CDU/CSU“ versus „übrige Parteien“ beziehungsweise alternativ Fraktionszugehörigkeit „bürgerliche Parteien“ versus „linke Parteien“). Allein die Variable zur differenzierten Messung der CDU/CSU-Abgeordneten im Vergleich zu den weiteren MdBs hatte ebenfalls einen signifikanten Einfluss auf die abhängige Variable: CDU/CSU-Politiker bewerten den Medieneinfluss negativer. Auf die Ergebnisse zu den in Tabelle 3 dargestellten Variablen hatte dies jedoch keine bemerkenswerten Auswirkungen.

37 CDU/CSU - Mittelwert: 1,86; SPD - Mittelwert: 2,03; FDP - Mittelwert: 1,37; Bündnis 90/ Die Grünen - Mittelwert: 1,69; Die Linke - Mittelwert: 2,79. 


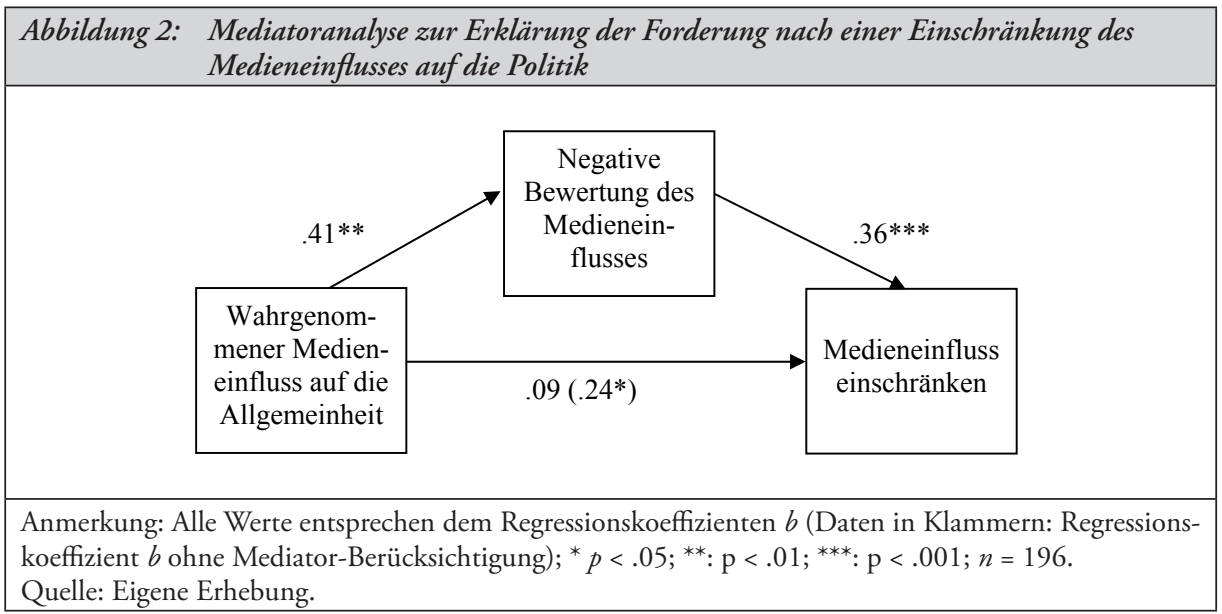

weg: Eine Mediatoranalyse ${ }^{38}$ bekräftigt die Vermutung, dass die Wahrnehmung eines großen Medieneinflusses dazu führt, den Medieneinfluss negativ zu bewerten. Je negativer wiederum diese Bewertung ausfällt, desto stärker wird der Aussage zugestimmt, der Medieneinfluss auf die Politik sollte eingeschränkt werden (siehe Abbildung 2). ${ }^{39}$

\section{Zuschreibung hohen Einflusses - mit indirekten Folgen}

Die befragten Bundestagsabgeordneten halten sich also selbst für wenig durch Medien beeinflussbar. Anderen Personen, insbesondere der Bevölkerung im Allgemeinen, wird jedoch eine sehr große politische Beeinflussbarkeit durch Medien unterstellt. Die Stärke des wahrgenommenen Medieneinflusses auf sich selbst hat bei den befragten Abgeordneten aber keine Auswirkungen auf die Bewertung dieses Einflusses. Je stärker allerdings die vermutete mediale Beeinflussung der Allgemeinheit ausfällt, desto negativer ist die Bewertung dieses Medieneinflusses. Und je negativer die Bewertung des Medieneinflusses, desto eher wird wenn auch in moderater Form - der Forderung zugestimmt, diesen Einfluss einzuschränken.

Die Annahmen der Theorien zu indirekten Medienwirkungen konnten also bestätigt werden: Auch in der Gruppe der Bundestagsabgeordneten bestehen Wahrnehmungsdifferenzen; und die Unterstellung eines Medieneinflusses auf andere hat Konsequenzen. Diese Resultate haben auch bei einer differenzierten Betrachtung unterschiedlicher Abgeordnetengruppen Bestand - mit der Einschränkung, dass die CDU/CSU-Abgeordneten etwas stärker als andere Politiker aus hohen Wirkungsvermutungen auf einen negativen Medieneinfluss schließen und in der Folge deutlicher eine Einschränkung dieses Einflusses befürworten.

38 Vgl. Kristopher J. Preacher / Andrew F. Hayes, SPSS and SAS Procedures for Estimating Indirect Effects in Simple Mediation Models, in: Behavior Research Methods, Instruments \& Computers, 36. Jg. (2004), H. 4, S. $717-731$.

39 Nach Fraktionszugehörigkeit getrennte Berechnungen verdeutlichen: Bei CDU/CSU-Abgeordneten ist der skizzierte indirekte Wirkungsweg im Vergleich zu den übrigen Abgeordneten etwas deutlicher ausgeprägt. 
In der Forschung etwa zur Mediatisierung der Politik wird häufig darauf verwiesen, dass Politiker einen starken medialen Druck auf ihre eigene Person verspüren und dieser Druck handlungsleitend für sie sein kann. ${ }^{40}$ Den Selbstauskünften der in dieser Studie befragten Abgeordneten zufolge werden die medialen Einflüsse indes nur als schwach wahrgenommen. ${ }^{41}$ Wichtiger erscheint aber, dass Medien auch auf indirektem Wege eine Wirkung auf politische Akteure ausüben können: Die befragten Bundestagsabgeordneten sind der Auffassung, dass das Bild von der Politik bei den Bürgern sehr stark von den Medien beeinflusst ist. Mit zunehmender Bedeutungszuschreibung steigt zunächst einmal auch die Skepsis gegenüber medialen Einflüssen. Die vorliegenden Daten veranschaulichen, dass eine solche kritische Sichtweise der medialen Bedeutung bei Weitem nicht allein von vermuteten Einflüssen auf die Bevölkerung geprägt wird. Eine Reihe weiterer Variablen, die in dieser Untersuchung nicht erfasst wurden, trägt zur Erklärung solcher Einflussbeurteilungen bei. Aber die vermuteten Medienwirkungen auf andere sind offensichtlich relevant für die Bewertung der so häufig beschworenen Macht der Medien - im Gegensatz zu den von den Abgeordneten eingeschätzten Wirkungen auf die eigene Person.

Die Skepsis gegenüber medialen Einflüssen hat schließlich weitere Folgen - im vorliegenden Fall für Forderungen nach einer Beschränkung dieses Einflusses. Das Item, mit dem die Forderung gemessen wurde, war eher vorsichtig formuliert, es wurde dort zum Beispiel kein Verbot bestimmter Medien(angebote) thematisiert. Außerdem war die absolute Zustimmung zu dem Item schwach. Allerdings ist es bemerkenswert, in welch deutlichem Maße sich offenbarte, dass vermutete und negativ bewertete Wirkungen auf andere die Einstellungen zu einer Einschränkung medialer Freiheit beeinflussen können.

Die beschriebene Wirkungskette mag plausibel sein, selbstverständlich ist sie keineswegs. Es wäre ebenso möglich gewesen, dass die Abgeordneten einen starken Medieneinfluss auf die Bevölkerung unterstellt und diesen Einfluss gerade deshalb positiv bewertet hätten. In diesem Fall hätten sich wohl auch keine Auswirkungen auf die Forderung gezeigt, den Medieneinfluss einzuschränken - im Gegenteil. Es muss überprüft werden, ob sich solche Ergebnisse zeigen, wenn der mediale Einfluss etwa in bestimmten Politikfeldern eingeschätzt und bewertet werden soll. So ergab eine vorherige Studie unter Politikern zum Beispiel, dass der Medieneinfluss im Bereich der Klimapolitik durchaus freundlicher beurteilt wurde. ${ }^{42}$

Einschränkend ist festzustellen, dass mit dem Forschungsdesign die hier gezogenen kausalen Schlüsse nicht zwangsläufig sind. Es wäre sogar eine umgekehrte Sichtweise auf die Resultate möglich: Bundestagsabgeordnete haben eine skeptische Sicht auf Medien, und in der Folge führt dies zu der Annahme (oder zu der Befürchtung), dass andere durch eben diese Medien beeinflusst werden könnten. Der theoretische Hintergrund sowie frühere Studien legen allerdings nahe, dass die Resultate in der im Text dargestellten Einflussrichtung interpretierbar sind. Außerdem wäre im umkehrten Fall zu erwarten gewesen, dass die CDU/CSU-Abgeordneten mit ihrer etwas kritischeren Betrachtung medialer Einflüsse auch eine stärkere Beeinflussung der Bevölkerung vermutet hätten. Gerade die Einschätzungen

40 Vgl. zum Beispiel Jesper Strömbeck, a.a.O. (Fn. 1).

41 Eine Erklärung hierfür könnte in der Formulierung des Items liegen, das zur Erfassung der Beeinflussung diente. Dort wurde allgemein gefragt, wie sehr das „Bild von der Politik“ von den Medien geprägt sei. In zukünftigen Studien müsste hier differenzierter erfasst werden, etwa durch Fragen nach medialen Einflüssen auf die eigene politische Arbeit.

42 Vgl. Marco Dohle / Gerhard Vowe, a.a.O. (Fn. 22). 
zur Intensität medialer Wirkungen unterscheiden sich jedoch zwischen den Fraktionen nicht.

Betont werden muss darüber hinaus, dass die Resultate trotz der relativ großen Stichprobe nicht bedenkenlos auf die Gesamtheit aller Abgeordneten übertragen werden können: Die Stichprobe der Untersuchung ist hinsichtlich der Fraktionszugehörigkeit verzerrt, CDU/ CSU-Abgeordnete beziehungsweise Vertreter der bürgerlichen Parteien sind überrepräsentiert. Dies wurde zumindest in Teilen berücksichtigt, indem die Parteizugehörigkeit kontrolliert oder differenzierte Analysen vorgenommen wurden. In zukünftigen Untersuchungen sollte jedoch geprüft werden, ob diese Resultate auch bei modifizierten Stichproben ihre Gültigkeit behalten. Auch sollten weitere Messungen vorgenommen werden: So ist bekannt, dass Individuen die mediale Berichterstattung gerade dann als zu ihren Ungunsten verzerrt wahrnehmen, wenn sie selbst eine eindeutige Meinung in einem Sachverhalt oder einem Konflikt haben. ${ }^{43}$ Dies könnte dazu führen, dass etwa CDU/CSU-Abgeordnete die Mehrheit an Medienberichten als eher links empfinden, Abgeordnete der Fraktion der Linken dagegen beispielsweise der Auffassung sind, in den Medien würden zumeist konservative Positionen vertreten. Diese Ansichten sind umso deutlicher ausgeprägt, je stärker die Zugehörigkeit zu einer Gruppierung und die Zustimmung zu deren Positionen sind. Und diese Ansichten haben wiederum Auswirkungen auf vermutete Medieneinflüsse. ${ }^{44}$ Es ist also möglich, dass sich hinter der reinen Parteizugehörigkeit weitere Variablen verbergen - etwa die individuell möglicherweise unterschiedlich ausgeprägte Bindung zur eigenen Partei -, die sich auf die Wahrnehmung von Medienberichten und Medieneinflüssen auswirken.

Politisches Handeln wird maßgeblich davon bestimmt, was die Politiker darüber denken, wie die Bevölkerung denkt. In vielerlei Hinsicht versucht die Politik daher systematisch, die Meinungsbilder der Wähler zu erfassen. Dazu bedient sie sich zum Beispiel der Demoskopie. Für das Ausmaß der Beeinflussbarkeit der Bürger - im vorliegenden Fall durch Medien liegen solche Daten jedoch nicht in exakter und verlässlicher Weise vor. Sie können daher von Politikern nur vermutet werden, zum Beispiel auf Basis ihrer Kenntnisse der Mediennutzung der Bevölkerung. Auch daher erscheint es notwendig, in weiteren Studien die Einflussvermutungen unter Bundestagsabgeordneten und anderen politisch tätigen Personen zu untersuchen - mit Blick auf die Intensität solcher wahrgenommenen Medieneinflüsse, aber auch hinsichtlich der Folgen für individuelles politisches Handeln.

43 Dieses Phänomen wird als Hostile-Media-Effekt bezeichnet und konnte in zahlreichen Studien bestätigt werden: Vgl. Robert P. Vallone / Lee Ross / Mark R. Lepper, The Hostile Media Phenomenon: Biased Perception and Perceptions of Media Bias in Coverage of the Beirut Massacre, in: Journal of Personality and Social Psychology, 49. Jg. (1985), H. 3, S. $577-585$.

44 Vgl. Jounghwa Choi / Myengja Yang / Jeongheon JC Chang, Elaboration of the Hostile Media Phenomenon. The Roles of Involvement, Media Scepticism, Congruency of Perceived Media Influence, and Perceived Opinion Climate, in: Communication Research, 36. Jg. (2009), H. 1, S. 54 -75 . 